\title{
Candida parapsilosis: principal causa de candidemia en un hospital de referencia para adultos de Costa Rica
}

\author{
Juan M. Villalobos, José A. Castro, Álvaro Avilés, M. Claudia Peláez, Teresita Somogyi y Lilliana Sandoval
}

\section{Candida parapsilosis: a major cause of bloodstream infection in a tertiary care hospital in Costa Rica}

Background: Invasive Candida bloodstream infections are frequent and display high mortality in clinical practice. There is scarce published on this topic in Central America. Objective: To characterize the epidemiology of candidemia in a hospital setting in Costa Rica. Methods: 210 cases of nosocomial candidemia were analyzed in patients over 17 years of age, admitted to Hospital Mexico, between 2007 and 2011. Descriptive and temporary analyses were performed and the risk factors associated with C. parapsilosis and survival were evaluated. Results: The incidence rate of candidemia was 1.47 cases per 1,000 admissions. The non-albicans Candida represented $62 \%$ of the isolated yeasts. Except for 2009, C. parapsilosis was the most commonly isolated species in four out of the five years reviewed, followed by $C$. albicans. There was a strong association between $C$. parapsilosis, the presence of a central venous catheter (OR: 4.8, CI 95\%: 1.8-14.6, p < 0.001) and the use of parenteral nutrition (p: 0.008). The 30-day mortality was 50\%. Candida albicans displayed the highest mortality and C. parapsilosis the lowest. Patients who did not receive anti-fungal treatment showed a significantly higher probability of death. Conclusions: The high incidence of candidemia from C. parapsilosis is directly related to the use of central venous catheters and parenteral nutrition. There is a need for creating local guidelines addressing the use of central venous catheters and parenteral nutrition, as well as implementing hand hygiene protocols.

Key words: Candida parapsilosis, candidemia, fungemia, sepsis, systemic inflammatory response syndrome.

Palabras clave: Candida parapsilosis, candidemia, fungemia, sepsis, síndrome de respuesta inflamatoria sistémica.

\section{Introducción}

L a candidemia continúa siendo la infección fúngica invasora más frecuente, representando cerca de 15 a $20 \%$ de todas las infecciones sanguíneas en los hospitales de los países desarrollados ${ }^{1-3}$.

Esta infección produce una estancia hospitalaria prolongada, un aumento en los costos de atención hospitalaria y una alta mortalidad atribuible ${ }^{4-7}$.

La incidencia reportada internacionalmente fluctúa entre 0,17 y 3,7 casos/1.000 egresos, siendo baja en los países desarrollados como Estados Unidos de América (E.U.A.) $\left(0,28-0,96\right.$ casos $/ 1.000$ egresos) y Europa $^{7}(0,20$ a 0,38 casos $/ 1.000$ egresos), mientras en América Latina es elevada $(0,33 \text { a } 2,49 \text { casos/1.000 egresos })^{8-10}$.

En tanto, la población con factores de riesgo para desarrollar esta complicación infecciosa continúa en crecimiento; en consecuencia el número de episodios que ocurren fuera de cuidados intensivos también ha aumentado, particularmente en servicios quirúrgicos ${ }^{11,12}$.

La distribución de las especies que causan candidemia ha cambiado en las últimas dos décadas; se ha observado un descenso de la frecuencia de Candida albicans, desde $90 \%$ en los años 70 hasta $50 \%$ o menos en la actualidad, en favor del aumento de las especies no albicans, tales como C. glabrata, C. tropicalis, C. parapsilosis y C. krusei ${ }^{13,14}$. La incidencia de candidemia por $C$. parapsilosis ha crecido progresivamente y estudios recientes la ubican como la tercera causa más frecuente de candidemia ${ }^{15,16}$.

En América Latina, C. albicans es responsable de 40 a $50 \%$ de los episodios, $C$. parapsilosis y $C$. tropicalis contribuyen cada una con 20 a $25 \%$ de los casos y la participación de C. glabrata y C. krusei es baja ${ }^{10}$.

La mortalidad cruda de la candidemia en adultos es globalmente de $30-59 \%^{7,11,17}$; en Latinoamérica ${ }^{8,9}$ varía entre 41 y $54 \%$.

A pesar de la importancia creciente de esta patología, existe muy poca información publicada proveniente de Centroamérica, ${ }^{9}{ }^{18}$. El propósito de este estudio fue caracterizar la epidemiología de la candidemia en uno de los hospitales de referencia de la Seguridad Social de Costa Rica, con el fin de ampliar el conocimiento existente sobre el comportamiento y la dinámica de dicha infección en esta región del mundo.
Caja Costarricense del Seguro Social. Hospital México. San José, Costa Rica.

Servicio de Infectología (JMV, AA, $\mathrm{MCP}$ ).

Dirección Farmacoepidemiología (JAC).

Laboratorio Clínico, División de Bacteriología (TS, LS).

Trabajo realizado en los Servicios de Infectología y Bacteriología del Hospital México, Caja Costarricense del Seguro Social, San José, Costa Rica.

Ninguno de los investigadores tiene conflictos de interés que declarar Estudio financiado por los investigadores, sin financiamiento externo.

Recibido: 20 de agosto de 2015 Aceptado: 31 de diciembre de 2015

Correspondencia a: Juan Manuel Villalobos Vindas juanvillaloboscr@gmail.com 


\section{Métodos}

\section{Tipo de estudio}

Retrospectivo de serie temporal, de la incidencia de candidemia en el Hospital México de la Caja Costarricense de Seguro Social en San José, Costa Rica. Este es uno de los tres hospitales nacionales de referencia para población adulta, que consta con la mayoría de las especialidades médicas y quirúrgicas; posee 550 camas, 22 de las cuales son de cuidados intensivos.

\section{Población}

Se incluyó todo paciente sobre 17 años de edad, que durante el período comprendido entre enero de 2007 y diciembre de 2011 tuviera al menos un hemocultivo con Candida spp. Se analizó únicamente el primer episodio de candidemia detectado por paciente.

\section{Aislamiento e identificación de las especies}

Durante este período, el tipo de hemocultivo utilizado fue el BacT/ALERT ${ }^{\circledR}$ SA (bioMérieux, Inc. Durham, North Carolina, USA). Su procesamiento se hizo siguiendo las recomendaciones del fabricante; la incubación y detección de crecimiento se efectuó en el sistema BacT/ ALERT 3D ${ }^{\circledR}$. La identificación de las especies de Candida se determinó por medio de ChromID Candida ${ }^{\circledR}$ (bioMérieux, Inc. Durham, North Carolina, USA), Vitek $2{ }^{\circledR}$ (YST ID CARD bioMérieux, Inc. Durham, North Carolina, USA), y microcultivo en agar harina de maíz (Oxoid $\AA$, Basingstoke, Hampshire, England). En los casos donde existió alguna incongruencia en la identificación de la especie, se utilizó el API 32C Candida System (bioMérieux, Inc. Durham, North Carolina, USA).

\section{Recolección de información}

La investigación se inició obteniendo el listado de todos los hemocultivos reportados como positivos por cualquier especie de Candida durante este período, de la base de datos del programa Observa ${ }^{\circledR}$ del equipo de identificación automatizada VITEK ${ }^{\circledR} 2$ (bioMérieux) en la División de Bacteriología del Laboratorio Clínico.

A partir de esta información, se procedió a la revisión de los expedientes clínicos, de donde se recolectaron las siguientes variables: sexo, edad, fecha de admisión al hospital, área de hospitalización, uso de catéter venoso central (CVC), días-catéter hasta el momento de obtener el(los) hemocultivo(s), uso simultáneo de nutrición parenteral, ventilación mecánica asistida al momento del diagnóstico de la candidemia, tratamiento antifúngico prescrito e inicio del mismo en relación al hemocultivo positivo, y los fallecimientos en los primeros 7 días (mortalidad temprana) y 30 días (mortalidad tardía) posteriores a la detección de la candidemia. Se determinó el índice de co-morbilidad de Charlson ${ }^{19,20}$ (ICC) ajustado por edad para cada paciente.
Un episodio de candidemia se clasificó como nosocomial si se desarrolló después de $72 \mathrm{~h}$ de hospitalización y no se estaba incubando al momento de la admisión; también se consideró como nosocomial todos los eventos ocurridos en los primeros 15 días posteriores a un egreso hospitalario.

Se excluyeron del estudio los casos donde no se pudo localizar el expediente clínico.

\section{Análisis de la información}

La incidencia acumulada por año se calculó por cada 1.000 egresos y se graficó su comportamiento en el tiempo y por especie. Las variables cuantitativas se describieron utilizando medidas de tendencia central y de dispersión. La comparación de los promedios se realizó por medio de análisis de variancia, y empleo la prueba de Kruskal-Wallis cuando no era válido aplicar el análisis de variancia. Las variables cualitativas se describieron por medio de distribuciones de frecuencia; además, se calcularon los intervalos de confianza al $95 \%$ para las frecuencias relativas y se compararon las proporciones utilizando pruebas de hipótesis (prueba de t-Student) por aproximación normal. Se estableció un nivel de significancia de 0,05 .

Se analizaron los posibles factores asociados a la presencia de C. parapsilosis en relación con las otras especies, utilizando para éstos el cálculo de odds ratio (OR) con su respectivos intervalos de confianza al $95 \%$ (IC 95\%). Además, se realizó un análisis de Kaplan-Meier y se aplicó una regresión de Cox para evaluar los factores relacionados con una mayor o menor sobrevida a 30 días después de detectada la presencia de Candida en sangre.

Para la realización del análisis estadístico se utilizaron los siguientes programas de cómputo: Microsoft Office Excel versión 2010 y SPSS versión 18.

Este estudio fue revisado y aprobado por el Comité Local de Bioética del Hospital México.

\section{Resultados}

Se analizaron 210 episodios distintos de candidemia y se excluyeron 10 por falta de información o no tener acceso al expediente clínico.

Todos los episodios fueron de adquisición nosocomial. Por año ocurrieron en promedio 42 episodios distintos de candidemia (28 a 70/año), para una incidencia acumulada de 1,47 casos por cada 1.000 pacientes egresados. Como se muestra en la Figura 1, se observó un incremento significativo de la incidencia a partir del año $2010(\mathrm{p}<0,0001)$ cuando el riesgo de padecer una candidemia aumentó en 2,15 veces (IC 95\% 1,63 a 2,82).

Este cambio en la incidencia de candidemia se debió principalmente a un mayor número de casos de infección por C. parapsilosis y C. albicans; el riesgo de enfermar 
por estas especies incrementó en 2,76 veces (IC 95\% 1,79 a 4,25) y 2,4 veces (IC 95\% 1,53 a 3,77), respectivamente.

Candida parapsilosis fue la especie más frecuentemente documentada (42\%), seguida por C. albicans (38\%), $C$. tropicalis $(10 \%)$ y C. glabrata $(7 \%)$; las demás especies representaron $3 \%$ (Tabla 1). Candida parapsilosis fue la especie más frecuente en 4 de los 5 años estudiados (Figura 1), la excepción ocurrió en el 2009, donde la frecuencia de $C$. parapsilopsis disminuyó y fue desplazada por $C$. albicans. La incidencia de las otras especies aumentó en los últimos años del estudio (Figura 1). Agrupadas todas las especies de Candida no albicans, constituyeron $62 \%$ de las causantes de candidemia.

El rango de edad fue 18-89 años con un promedio de 54 años y una mediana de 58 años. La incidencia de candidemia por edad fue $0,78 / 1.000$ egresos bajo 40 años de edad, 1,97 en el grupo de 40 a 69 años y 2,41 en los de 70 y más años. No existió diferencia estadísticamente significativa entre la edad promedio de los pacientes y la especie involucrada (Tabla 1).

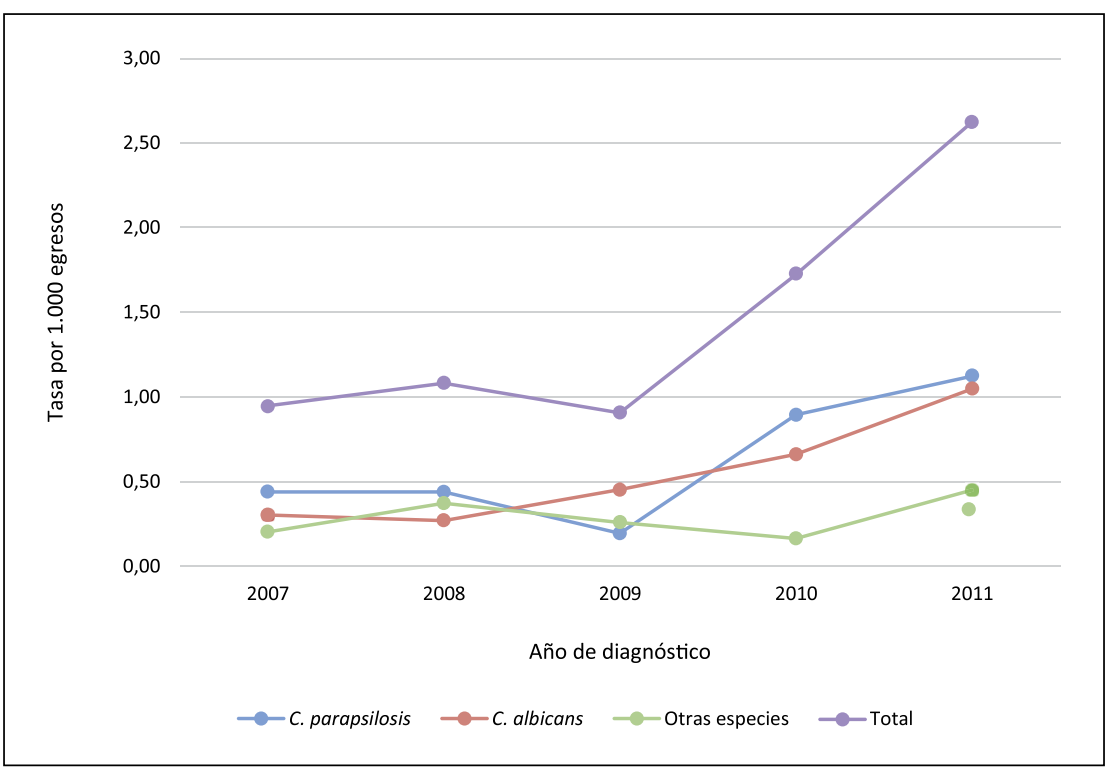

Figura 1. Incidencia acumulada de candidemia por 1.000 egresos, según año y especie.

\begin{tabular}{|c|c|c|c|c|c|c|}
\hline Variable & $\begin{array}{l}\text { C. parapsilosis } \\
\text { (n: } 89)\end{array}$ & $\begin{array}{l}\text { C. albicans } \\
\text { (n: 79) }\end{array}$ & $\begin{array}{l}\text { C. glabrata } \\
\text { (n: 15) }\end{array}$ & $\begin{array}{l}\text { C. tropicalis } \\
\text { (n: 20) }\end{array}$ & $\begin{array}{l}\text { Otras especies } \\
\quad(\mathrm{n}: 7)\end{array}$ & $\begin{array}{l}\text { Total } \\
\text { (n: } 210)\end{array}$ \\
\hline Sexo masculino $\mathrm{n}(\%)$ & $46(52)$ & $36(46)$ & $9(60)$ & $13(65)$ & $3(43)$ & $107(51)$ \\
\hline Promedio de edad (años) (IC 95\%) & $52,0(48,8-56,1)$ & $56,8(52,6-61,0)$ & $58,0(48,2-67,8)$ & $50,0(42,5-57,6)$ & $53,9(34,1-73,8)$ & $54,1(51,6-56,7)$ \\
\hline \multicolumn{7}{|l|}{ Grupos de edad n (\%) } \\
\hline 12-39 años & $24(27)$ & $17(22)$ & $3(20)$ & $7(35)$ & $2(29)$ & $53(25)$ \\
\hline 40 a 69 años & $48(54)$ & $40(51)$ & $8(53)$ & $10(50)$ & $3(43)$ & $109(52)$ \\
\hline 70 años y más & $17(19)$ & $22(28)$ & $4(27)$ & $3(15)$ & $2(29)$ & $48(23)$ \\
\hline $\begin{array}{l}\text { Promedio de días de hospitalización hasta la candidemia } \\
\text { (IC 95\%) }\end{array}$ & $27,4(21,6-33,1)$ & $22,0(17,8-26,1)$ & $36,7(3,8-69,6)$ & $18,9(12,4-25,3)$ & $30,4(9,8-51,0)$ & $25,3(21,6-29,0)$ \\
\hline \multicolumn{7}{|l|}{ n de pacientes con: } \\
\hline Índice de co-morbilidad de Charlson $\geq 3$ (\%) & $36(41)$ & $46(59)$ & $9(64)$ & $9(45)$ & $4(57)$ & $104(50)$ \\
\hline Catéter venoso central (CVC) (\%) & $84(94)$ & $60(76)$ & $12(80)$ & $16(80)$ & $6(86)$ & $178(85)$ \\
\hline Promedio de días con CVC hasta la candidemia (IC 95\%) & $11,0(8,5-13,5)$ & $10,4(9,0-11,8)$ & $10,2(6,9-13,4)$ & $11,0(6,7-15,3)$ & $11,7(6,2-17,2)$ & $10,8(9,4-12,1)$ \\
\hline Nutrición parenteral n (\%) & $49(55)$ & $31(39)$ & $5(33)$ & $5(25)$ & $3(43)$ & $93(44)$ \\
\hline Ventilación mecánica asistida n (\%) & $45(51)$ & $26(33)$ & $7(47)$ & $5(25)$ & $3(43)$ & $86(41)$ \\
\hline Candida spp en otro sitio n (\%) & $12(13)$ & $22(28)$ & $6(40)$ & $4(20)$ & $0(0)$ & $44(21)$ \\
\hline \multicolumn{7}{|l|}{ n de pacientes*: } \\
\hline Sin tratamiento $(\%)$ & $15(17)$ & $16(20)$ & $4(27)$ & $3(15)$ & $1(14)$ & $39(19)$ \\
\hline \multicolumn{7}{|l|}{ En tratamiento con: } \\
\hline Anfotericina (\%) & $39(44)$ & $36(46)$ & $6(40)$ & $13(65)$ & $5(71)$ & $99(47)$ \\
\hline Caspofungina (\%) & $12(13)$ & $10(13)$ & $3(20)$ & $2(10)$ & $0(0)$ & $27(13)$ \\
\hline Fluconazol (\%) & $18(20)$ & $16(16)$ & $2(13)$ & $2(10)$ & $0(0)$ & $38(18)$ \\
\hline Sin especificar (\%) & $5(6)$ & $1(1)$ & $0(0)$ & $0(0)$ & $1(1)$ & $7(3)$ \\
\hline \multicolumn{7}{|l|}{ Mortalidad } \\
\hline Temprana (a 7 días) & $11(12)$ & $25(32)$ & $4(27)$ & $4(20)$ & $1(14)$ & $45(21)$ \\
\hline Tardía (a 30 días) & $34(38)$ & $50(63)$ & $6(40)$ & $10(50)$ & $5(71)$ & $105(50)$ \\
\hline
\end{tabular}




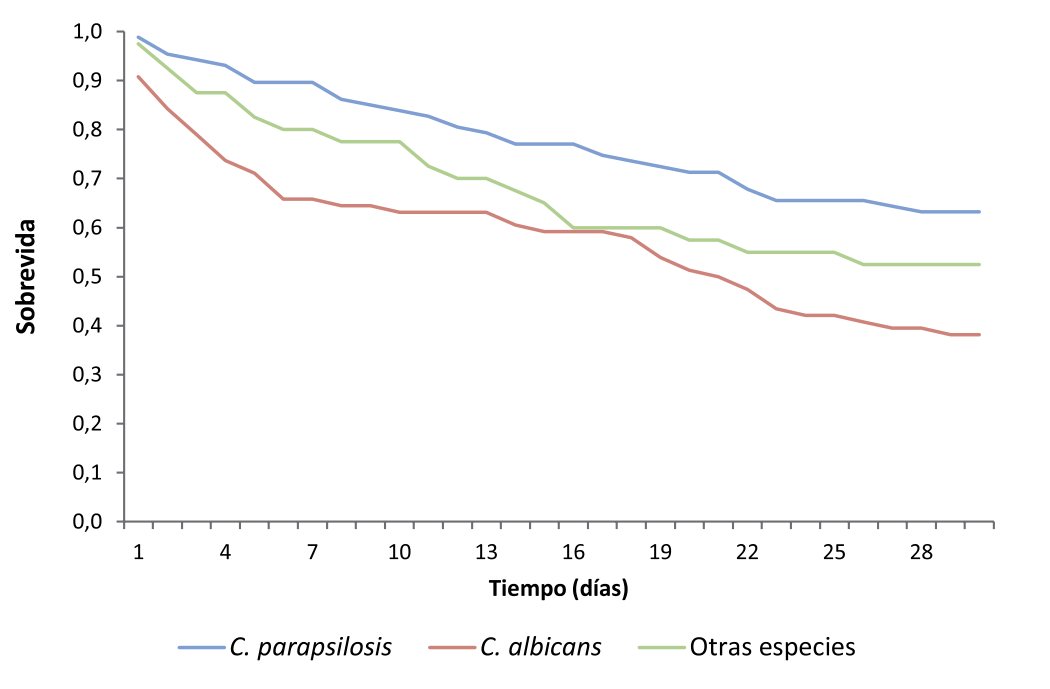

Figura 2. Estimación de Kaplan-Meier de la sobrevida en pacientes con candidemia, según especie.

Cuarenta y seis por ciento de los pacientes estuvieron hospitalizados en las especialidades quirúrgicas; las especialidades médicas aportaron $38 \%$ de los casos y cuidados intensivos sólo 4\%, presentando este último una tasa de candidemia de 8,5 casos por cada 1.000 pacientes admitidos. El 12\% restante de los pacientes fueron detectados en los otros servicios del hospital.

La media de días de hospitalización hasta el momento de la candidemia fue de 25 días, sin que se apreciara una diferencia estadísticamente significativa entre las especies (Tabla 1). Cuando esto se evaluó por servicio de hospitalización, el promedio de estancia en las especialidades quirúrgicas fue mayor (32 días) que en las especialidades médicas (19 días) o cuidados intensivos (20 días), diferencia que fue estadísticamente significativa (p: 0,01).

Al evaluar la co-morbilidad asociada de los pacientes, se determinó que 50,5\% de ellos poseía un ICC de 3 o más puntos (sobrevida estimada a 10 años de $77 \%$ o menos). El $64,3 \%$ de los pacientes con $C$. glabrata tuvo un ICC mayor o igual que 3 , mientras que en los pacientes con C. parapsilosis este porcentaje fue $41,4 \%$ (Tabla 1). Los pacientes presentaron una gran diversidad de condiciones patológicas; las más frecuentes fueron: cáncer (29\%), fístula intestinal post-quirúrgica (23\%), diabetes mellitus $(25 \%)$ e insuficiencia renal crónica $(5 \%)$. Los pacientes con C. glabrata presentaron una mayor frecuencia de diabetes mellitus.

Ochenta y cinco por ciento de los pacientes tenía un CVC al momento de la candidemia, con un promedio de duración de 11 días (IC 95\%: 9,4 a 12,1).

En los casos de C. parapsilosis la frecuencia de CVC fue la más alta (94\%), mientras que para las demás espe- cies varió entre 76 y $86 \%$ (Tabla 1 ). Se observó una fuerte asociación entre la presencia de CVC y candidemia por $C$. parapsilosis en comparación con las otras especies (OR: 4,8, IC 95\%: 1,8 a 14,6, p <0,001). Al estudiar el número de días entre la colocación del CVC y el diagnóstico de la candidemia, no se encontró una diferencia significativa entre las especies (Tabla 1).

Cuarenta y cuatro por ciento de los pacientes estaba recibiendo nutrición parenteral al momento de la candidemia (Tabla 1) y se detectó una mayor probabilidad de que la especie asociada fuera C. parapsilosis (OR: 2,1, IC 95\% 1,2 a 3,8, p: 0,008).

En relación con el tratamiento de la candidemia, 19\% de los pacientes no recibió tratamiento antifúngico. El medicamento más utilizado fue anfotericina B (60\%), seguido por fluconazol (23\%) y caspofungina (17\%); no existió diferencia en la selección del antifúngico por especie (Tabla 1). A la mayoría de los pacientes (82\%) se les inició el tratamiento antifúngico 24 h o más después de tomado el hemocultivo que permitió el diagnóstico (promedio 2,3 $\pm 2,4$ días, rango $-5 \mathrm{a}+12$ días). Quienes no recibieron tratamiento antifúngico $(18 \%)$ tuvieron un riesgo significativamente mayor de fallecer a los 30 días, cuando se compararon con los pacientes que sí recibieron antifúngicos (HR: 2,9, IC 95\% 1,8 a 4,6, p < $0,001)$. De los pacientes que no recibieron tratamiento, $50 \%$ fallecieron en los tres días posteriores al reporte del hemocultivo positivo.

La mortalidad a 30 días fue de 50\%; la especie con la menor mortalidad fue C. parapsilosis (38\%) (Figura 2). Por el contrario, C. albicans presentó la más alta mortalidad (63\%). Mediante la regresión de Cox se encontró que los pacientes con C. parapsilosis tenían una menor probabilidad de morir en relación con las otras especies (HR: 0,6, IC 95\% 0,4 a 0,9, p: 0,020) (Figura 2). Por otra parte, factores como el sexo femenino, la edad mayor a 70 años, la presencia de cáncer y ventilación mecánica asistida presentaron una asociación positiva y estadísticamente significativa con la mortalidad a 30 días.

\section{Discusión}

Este estudio muestra las características epidemiológicas y clínicas más sobresalientes de la candidemia en un hospital de referencia de Costa Rica. La tasa de candidemia en este hospital está dentro del rango reportado en la literatura médica $(0,17 \text { a } 3,7 \text { casos } / 1.000 \text { ingresos })^{7}$, es similar a otros hospitales latinoamericanos ${ }^{8,9}$ pero mayor a lo reportado en hospitales de países desarrollados ${ }^{7,21}$.

Contrariamente a lo descrito por otros investigadores $^{20-22}$ la mayoría de nuestros casos procedían de especialidades quirúrgicas y no de cuidados intensivos, esto posiblemente relacionado con complicaciones post- 
quirúrgicas de alto riesgo para desarrollar candidemia como la fístula intestinal que estaba presente en $23 \%$ de los pacientes. Además, muchos de estos pacientes de alto riesgo fueron atendidos en salas generales, debido a las pocas camas (4\%) asignadas a cuidados intensivos en comparación con el tamaño y la complejidad del hospital.

El predominio de las especies no albicans como causa de candidemia en nuestro hospital es similar a lo descrito en otros centros de América Latina ${ }^{8,9,24}$ y del mundo ${ }^{25-27}$. Sin embargo, es sorprendente que $C$. parapsilosis sea la causa más frecuente de candidemia, lo que no parece deberse a un brote aislado, pues fue la especie predominante en cuatro de los cinco años estudiados. Este comportamiento sugiere que se trata de un problema de salud endémico y difiere de la mayoría de los reportes de la literatura científica, donde C. albicans es la especie más frecuente y $C$. parapsilosis ocupa el segundo o tercer lugar con una frecuencia que oscila entre 10 y $25 \%{ }^{11,26,28}$. En América Latina, alcanza a $18-25 \%^{8-10}$, pero sin sobrepasar a C. albicans. Reportes esporádicos en la literatura internacional coinciden al respecto con lo descrito en este estudio ${ }^{29-32}$ pero analizan series pediátricas o bien, representan brotes aislados en servicios específicos de un hospital.

Nuestros datos indican que la puerta de entrada más importante al torrente sanguíneo de $C$. parapsilosis es la presencia de un CVC, pues se estableció una fuerte asociación entre ambos, observación que coincide con lo publicado por otros investigadores ${ }^{29,33,34}$. Lo anterior se explica, al menos parcialmente, por la capacidad de esta especie de adherirse a los materiales sintéticos y formar una placa de crecimiento (bio-película) en los $\mathrm{CVC}^{35-38}$. Adicionalmente, la composición de las soluciones de nutrición parenteral que se infunden por los $\mathrm{CVC}$ favorece el crecimiento de esta especie y, por consecuencia, facilita su invasión sanguínea ${ }^{39,40}$, lo que podría explicar la relación estadísticamente significativa entre $C$. parapsilosis y el uso de la nutrición parenteral como se demuestra en este estudio.

¿Cómo alcanza C. parapsilosis el CVC de estos pacientes? Es una interrogante a la cual no podemos dar respuesta con los datos obtenidos; sin embargo, como ha sido descrito en la literatura científica ${ }^{30,40}$, el mecanismo más importante involucra las manos del personal de salud que atiende a estos pacientes. El estudio realizado por Carrillo-Dover y cols. ${ }^{41}$, en otro centro costarricense de referencia para adultos muy similar al nuestro, también sugiere que ésta sea la vía implicada en nuestro medio.

La mayoría de los pacientes fueron tratados con los antifúngicos recomendados por las guías internacionales de esa época; sin embargo, a pesar del excelente perfil terapéutico de caspofungina, fue el fármaco menos prescrito, posiblemente debido a su alto precio cuando se le compara con otros antifúngicos ${ }^{42,43}$.
La mortalidad a 30 días encontrada en este estudio es alta si la comparamos con la mayor serie latinoamericana reportada ${ }^{9}$ con lo descrito para los países desarrollados ${ }^{7}$. Cuarenta y tres por ciento de las defunciones ocurrieron en los primeros siete días, lo que podría estar relacionado con los siguientes elementos: una quinta parte de los pacientes no recibió tratamiento (19\%); además, la gran mayoría de los pacientes $(82 \%)$ inició tratamiento antifúngico en 2,3 días promedio después de tomado el hemocultivo diagnóstico, lo que generó una demora importante en el inicio del mismo y se ha demostrado que este retraso influye negativamente en la sobrevida de los pacientes ${ }^{44-47}$. Estos resultados son muy similares a lo descrito para Latinoamérica por Nucci y cols 9 .

Una de las debilidades más importantes de este estudio fue no contar con una separación molecular de las especies del "complejo psilosis" lo que limitó determinar cuál de las tres especies de este grupo es la dominante, aunque lo publicado ${ }^{48-50}$ señala a $C$. parapsilosis sensu stricto como la más importante. La falta de estudios genotípicos impidió definir la presencia de genotipos nosocomiales responsables del predominio de C. parapsilosis sobre las demás especies ${ }^{51}$.

En conclusión, este estudio muestra que durante el período analizado existió un predominio de la especie $C$. parapsilosis como causa de candidemia en el Hospital México y la señala como un problema endémico en este centro. La relación establecida entre el CVC, la nutrición parenteral y $C$. parapsilosis, señala que es necesario establecer guías locales para la colocación, el uso y manipulación adecuada del CVC, así como el uso racional de la nutrición parenteral. Tales estrategias podrían ser claves para reducir la alta incidencia de esta infección.

Sin duda, existe al menos otro factor crítico que debe estar participando en esta dinámica: las manos del personal que atiende a estos pacientes. En consecuencia, implementar las medidas necesarias para mejorar la higiene de las manos representa una gran oportunidad para modificar la frecuencia de esta especie.

Una alta sospecha clínica y un inicio más temprano o quizás anticipado del tratamiento antifúngico representan las mejores alternativas para cambiar la alta mortalidad de esta infección en nuestro hospital.

Finalmente, es imprescindible realizar un estudio multicéntrico para definir mejor la situación de esta patología infecciosa dentro del sistema de seguridad social costarricense.

\section{Resumen}

Introducción: Las infecciones invasoras por Candida son frecuentes y de alta mortalidad. Existe poca información publicada de la región centroamericana. Objetivo: 
Caracterizar la epidemiología de la candidemia en un hospital de Costa Rica. Métodos: Se analizaron 210 episodios de candidemia nosocomial en pacientes sobre 17 años de edad, entre los años 2007 y 2011. Se realizó un análisis descriptivo y temporal de la serie y evaluación de las características clínicas asociadas haciendo énfasis en C. parapsilosis. Resultados: La incidencia acumulada de candidemia fue 1,47 casos/1.000 admisiones. Las especies de Candida no albicans constituyeron $62 \%$ de las levaduras aisladas. Exceptuando el año 2009, $C$. parapsilosis fue la especie predominante en cuatro de los cinco años estudiados, seguida por C. albicans. Se demostró una fuerte asociación entre $C$. parapsilosis, la presencia de catéter venoso central (OR: 4,8, IC 95\%: $1,8-14,6, \mathrm{p}<0,001)$ y el uso de nutrición parenteral (p: 0,008). La mortalidad a 30 días fue de 50\%. Candida albicans mostró la mortalidad más alta y $C$. parapsilosis la más baja. Los pacientes que no recibieron tratamiento antifúngico presentaron un aumento significativo en la mortalidad. Conclusiones: La incidencia elevada de candidemia por C. parapsilosis está relacionada con los catéteres venosos centrales y la administración de nutrición parenteral. Para su control es necesario establecer guías locales para uso de los catéteres venosos centrales y la nutrición parenteral, así como implementar estrategias para promocionar la higiene de las manos.

\section{Referencias bibliográficas}

1.- Wisplinghoff H, Bischoff T, Tallent S M, Seifert H, Wenzel R P, Edmond M B. Nosocomial bloodstream infections in US hospitals: analysis of 24,179 cases from a prospective nationwide surveillance study. Clin Infect Dis 2004; 39: 309-17.

2.- Pfaller M A, Diekema D J. Role of sentinel surveillance of candidemia: trends in species distribution and antifungal susceptibility. J Clin Microbiol 2002; 40: 3551-57.

3.- Alangaden G J. Nosocomial fungal infections epidemiology, infection control, and prevention. Infect Dis Clin North Am 2011; 25: 201-25.

4.- Zaoutis T E, Argon J, Chu J, Berlin J A, Walsh T J, Feudtner C, et al. The epidemiology and attributable outcomes of candidemia in adults and children hospitalized in the United States: a propensity analysis. Clin Infect Dis 2005; 41: 1232-9.

5.- Morgan J, Meltzer M I, Plikaytis B D, Sofair A N, Huie-White S, Wilcox S, et al. Excess mortality, hospital stay, and cost due to candidemia: a case-control study using data from population based candidemia surveillance. Infect Control Hosp Epidemiol 2005; 26: 540-7.

6.- Gagne J J, Goldfarb N I. Candidemia in the in-patient setting: treatment options and economics. Expert Opin Pharmacother 2007; 8: 1643-50.

7.- Eggimann P, Garbino J, Pittet D. Epidemiology of Candida species infections in critically ill non-immunosuppressed patients. Lancet Infect Dis 2003; 3: 685-702.

8.- Colombo A L, Nucci M, Park B J, Nouer S A, Arthington-Skaggs B, da Matta D A, et al. Epidemiology of candidemia in Brazil: a nationwide sentinel surveillance of candidemia in eleven medical centers. J Clin Microbiol 2006; 44: 2816-23.

9.- Nucci M, Queiroz-Telles F, Alvarado-Matute T, Tiraboschi I, Cortés J, Zurita J, et al. Epidemiology of candidemia in Latin America: a laboratory-based survey. PLoS ONE 2013; 8: e59373.

10.- Nucci M, Thompson-Moya L, GuzmánBlanco M, Tiraboschid I N, Cortese J A, Echevarría J, et al. Recomendaciones para el manejo de la candidemia en adultos en América Latina. Rev Iberoam Micol 2013; 30 (3S1): 179-88.

11.- Morgan J. Global trends in candidemia: review of reports from 1995-2005. Cur Infect Dis Rep 2005; 7: 429-39.

12.- Méan M, Marchet, Calandra T. Bench-tobedside review: Candida infections in the intensive care unit. Critical Care 2008; 12 : 204-13.

13.- Pfaller M A, Jones R N, Doren G V, Sader H S, Hollis R J, Messer S A, et al. International surveillance of bloodstream infections due to Candida species in the European SENTRY Program: species distribution and antifungal susceptibility including the investigational triazole and echinocandin agents. Diag Microbiol Infect Dis 1999; 35: 19-25.

14.- Guinea J. Global trends in the distribution of Candida species causing candidemia. Clin Microbiol Infect 2014; 20 (Suppl. 6): 5-10.

15.- Trofa D, Gácser A, Nosanchuk J D. Candida parapsilosis, an emerging fungal pathogen. Clin Microbiol Rev 2008; 21: 606-25.

16.- Pfaller M A, Diekema D J. Epidemiology of invasive candidiasis: a persistent public health problem. Clin Microbiol Rev 2007; 20: 133-63.

17.- Caballero E, Boza R, González K. Candidiasis sistémica-Experiencia en el Hospital San Juan de Dios 1996-1998. Rev Costarric Cienc Méd 1999; 20: 153-65.

18.- Charlson M E, Pompei P, Ales K L, MacKenzie C R. A new method of classifying prognostic comorbidity in longitudinal studies: development and validation. See comment in PubMed Commons belowJ Chronic Dis 1987; 4: 373-83.

19.- Quan H, Li B, Couris C M, Fushimi K, Graham P, Hider P, et al. Updating and validating the Charlson comorbidity index and score for risk adjustment in hospital discharge abstracts using data from 6 countries. Am J Epidemiol 2011; 173: 676-82.

20.- Tortorano A M, Kibbler C, Peman J, Bernhardt H, Klingspor L, Grillot R. Candidemia in Europe: epidemiology and resistance. International J Antimicrob Agents 2006; 27: 359-66.

21.- Eggimann P, Francioli P, Bille J, Schneider R, Wu M M, Chapuis G, et al. Fluconazole prophylaxis prevents intra-abdominal candidiasis in high risk surgical patients. Crit Care Med 1999; 27: 1066-72.

22.- Ostrosky-Zeichner L, Pappas P. Invasive candidiasis in the intensive care unit. Crit Care Med 2006; 34: 857-63.

23.- Colombo A L, Nucci M, Salomao R, Branchini M L, Richtmann R, Derossi A, et al. High rate of non albicans candidemia in Brazilian tertiary care hospitals. Diagn Microbiol Infect Dis 1999; 34: 281-6.

24.- Borges M, Zaragoza R. Critical overview of clinical guidelines relating to invasive fungal infections. International J Antimicrob Agents 2008; 32: S155-9.

25.- Pfaller M A, Diekema D J, Gibbs D L, Newell V A, Ng K P, Colombo A, et al. Geographic and temporal trends in isolation and antifungal susceptibility of Candida parapsilosis: a global assessment from the ARTEMIS DISK antifungal surveillance program, 2001 to 2005. J Clin Microbiol 2008; 46: 842-9.

26.- Calandra T, Marchetti O. Clinical trials of antifungal prophylaxis among patients undergoing surgery. Clin Infect Dis 2004; 39 : S185-92.

27.- Quindós G. Epidemiology of candidaemia and invasive candidiasis. A changing face. Rev Iberoam Micol 2014; 31: 42-8.

28.- Brito L R, Guimaraes T, Nucci M, Rosas R C, Almeida L P, da Matta D A, et al. Clinical and microbiological aspects of candidemia due to 
Candida parapsilosis in Brazilian tertiary care hospitals. Med Mycol 2006; 44: 261-6.

29.- Diekema D J, Pfaller M A. Nosocomial candidemia: an ounce of prevention is better than a pound of cure. Infect Control Hosp Epidemiol 2004; 25: 624-6.

30.- Dizbay M, Fidan I, Kalkanci A, Sari N, Yalcin B, Kustimur S, et al. High incidence of Candida parapsilosis candidaemia in nonneutropenic critically ill patients: Epidemiology and antifungal susceptibility. Scand J Infect Dis 2010; 42: 114-20.

31.- Huang Y C, Lin T Y, Leu H S, Peng H L, Wu J H, Chang H Y. Outbreak of Candida parapsilosis fungemia in neonatal intensive care units: clinical implications and genotyping analysis. Infection 1999; 27: 97-102.

32.- Levin A S, Costa S F, Mussi N S, Basso M, Sinto S I, Machado C, et al. Candida parapsilosis fungemia associated with implantable and semi-implantable central venous catheters and the hands of healthcare workers. Diagn Microbiol Infect Dis 1998; 30: 243-9.

33.- Medrano D J, Brilhante R S, Cordeiro R A, Rocha M F, Rabenhorst S H, Sidrim J J. Candidemia in a Brazilian hospital: the importance of Candida parapsilosis. Rev Inst Med Trop Sao Paulo 2006; 48: 17-20.

34.- Hawser S P, Douglas L J. Biofilm formation by Candida species on the surface of catheter materials in vitro. Infect Immun 1994; 62: 915-21.

35.- Branchini M L, Pfaller M A, Rhine-Chalberg J, Frempong T, Isenberg H D. Genotypic variation and slime production among blood and catheter isolates of Candida parapsilosis. J Clin Microbiol 1994; 32: 452-6.

36.- Ramage G, Saville S P, Thomas D P, López-Ribot J L. Candida biofilms: an update. Eukaryot Cell 2005; 4: 633-8.
37.- Silva $S$, Negri M, Henriques M, Oliveira R, Williams D W, Azeredo J. Adherence and biofilm formation of non-Candida albicans species. Trends Microbiol 2011; 19: 241-7.

38.- Weems J J Jr., Chamberland M E, Ward J, Willy M, Padhye A A, Solomon S L. Candida parapsilosis fungemia associated with parenteral nutrition and contaminated blood pressure transducers. J Clin Microbiol 1987; 25: 1029-32.

39.- Pfaller M A. Nosocomial candidiasis: emerging species, reservoirs, and modes of transmission. Clin Infect Dis 1996; 22: S89-S94.

40.- Carrillo-Dover P, Álvarez-Vega C, SalasCampos I, Mora-Brenes M. Aislamiento de Candida spp. y otras levaduras en el personal que labora en áreas críticas del Hospital San Juan de Dios. Acta Méd Costarric 2009; 51: 166-71.

41.- Pappas P G, Rex J H, Sobel J D, Filler S G, Dismukes W E, Walsh T J, et al. Guidelines for treatment of candidiasis. Clin Infect Dis 2004; 38: 161-89.

42.- Pappas P G, Kauffman C A, Andes D, Benjamin D K, Calandra T F, Edwards J E, et al. Clinical Practice Guidelines for the Management of Candidiasis: 2009 Update by the Infectious Diseases Society of America. Clin Infect Dis 2009; 48: 503-35.

43.- Kollef M, Micek S, Hampton N, Doherty J A, Kumar A. Septic shock attributed to Candida infection: importance of empiric therapy and source control. Clin Infect Dis 2012; 54: 1739-46.

44.- Morrell M, Fraser V J, Kollef M H. Delaying the empiric treatment of Candida bloodstream infection until obtaining positive blood culture results: A potential risk factor for hospital mortality. Antimicrob Agents Chemother 2005; 49: 3640-5.

45.- Garey K W, Rege M, Pai M P. Time to initiation of fluconazole therapy impacts mortality in patients with candidemia: A multicenter study. Clin Infect Dis 2006; 43: 25-31.

46.- Grim S A, Berger K, Teng C, Gupta S, Layden J E, Janda W M, et al. Timing of susceptibility-based antifungal drug administration in patients with Candida bloodstream infection: correlation with outcomes. J Antimicrob Chemother 2012; 67: 707-14.

47.- Cantón E, Pemán J, Quindós G, Eraso E, Miranda-Zapico I, Álvarez M, et al. Prospective multicenter study of the epidemiology, molecular identification, and antifungal susceptibility of Candida parapsilosis, Candida orthopsilosis, and Candida metapsilosis isolated from patients with candidemia. Antimicrob Agents Chemother 2011; 55: 5590-96.

48.- Lockhart S R, Messer S A, Pfaller M A, Diekema D J. Geographic distribution and antifungal susceptibility of the newly described species Candida orthopsilosis and Candida metapsilosis in comparison to the closely related species Candida parapsilosis. J Clin Microbiol 2008; 46: 2659-64.

49.- Gonçalves S S, Amorim C S, Nucci M, Padovan A C B, Briones M R S, Melo A S A, et al. Prevalence rates and antifungal susceptibility profiles of the Candida parapsilosis species complex: results from a nationwide surveillance of candidaemia in Brazil. Clin Microbiol Infect 2010; 16: 885-7.

50.- Escribano P, Marcos-Zambrano L J, Recio S, Rodríguez-Créixems R, Muñoz P, Bouza E. Genotypic analysis of Candida parapsilosis isolates causing fungemia: Evidence of endemic genotypes in the hospital. 23rd European Congress of Clinical Microbiology and Infectious Diseases, abstract (R-2708) 2013. 